\title{
The Clinical Implication of Dynamic Hematological Parameters in COVID-19: A Retrospective Study in Chongqing, China
}

\author{
Huan Yang (D) ${ }^{1,2}$ \\ Yuan $\mathrm{Xu}^{2}$ \\ Zhijie $\mathrm{Li}^{2}$ \\ Ling Yan ${ }^{2}$ \\ Jing Wang ${ }^{3}$ \\ Pu Liao ${ }^{1,2}$ \\ 'School of Clinical Medicine, Southwest \\ Medical University, Luzhou, People's \\ Republic of China; ${ }^{2}$ Department of \\ Clinical Laboratory, Chongqing General \\ Hospital, Chonging, People's Republic of \\ China; ${ }^{3}$ Department of Clinical \\ Laboratory, Chongqing Public Health \\ Medical Center, Chonging, People's \\ Republic of China
}

Correspondence: Pu Liao; Jing Wang Email liaopu@ucas.ac.cn;

4034I3860@qq.com
Purpose: To analyze the clinical characteristics of patients with coronavirus disease 19 (COVID-19) in Chongqing, and identify the potential hematological markers for reference.

Patients and Methods: 78 COVID-19-infected patients in Chongqing were recruited and divided into the non-severe and the severe group. The clinical characteristics and hematological features of the patients of the two groups were compared. Receiver-operating characteristic curves (ROC) were calculated to evaluate the diagnostic performance of potential markers, and the dynamic changes of blood routine analyzing items were compared between the non-severe and severe groups.

Results: 78 patients (median age of 45 years, 41 females and 37 males) were enrolled. The patients in the severe group exhibited significantly lower lymphocyte $(\mathrm{P}<0.05)$ but higher neutrophil to lymphocyte ratio $(\mathrm{NLR})(\mathrm{P}<0.05)$ than the patients in the non-severe group. The highest area under the ROC curve (AUC) was lymphocyte (0.74). The patients in the severe group had a lower level of lymphocyte during hospitalization $(\mathrm{P}<0.01)$ and lymphocyte-monocyte ratio (LMR) in the progressive and convalescent phases $(\mathrm{P}<0.05)$ than the patients in the non-severe group. However, the level of neutrophil of the patients in the severe group was higher in the progressive phase $(\mathrm{P}<0.05)$, and so was NLR in the acute, progressive, and convalescent-phase $(\mathrm{P}<0.05)$.

Conclusion: Infected with COVID-19 changed the levels of lymphocyte, neutrophil, LMR, and NLR in the blood, and these analyzing items were significantly different between the non-severe and severe groups. Furthermore, the dynamic changes of lymphocyte and NLR levels may help discriminate the severe group from the non-severe group.

Keywords: COVID-19, lymphocyte, neutrophil, neutrophil-lymphocyte ratio

\section{Introduction}

The emergence of COVID-19 marked the third time that a highly pathogenic coronavirus has been introduced to humans in the 21 st century. ${ }^{1}$ Globally, as of 6:00 pm, 15 July 2021, there have been over 188,128,952 confirmed cases of COVID-19, including 4,059,339 deaths (2.2\% mortality), reported by the World Health Organization (WHO). Severe Acute Respiratory Syndrome Coronavirus 2 (SARS-CoV-2) resulted in enormous loss of life and serious economic burden in the affected areas. At present, the majority of previous studies on the clinical and hematological parameters of COVID-19-infected patients at admission did not receive drug treatment, ${ }^{2-7}$ and there were few studies on dynamic changes with the patient's disease course. ${ }^{8,9}$ 
Several hematological changes were reported in COVID19 patients, including leukocytosis, decreased lymphocyte count, elevated levels of D-dimer, neutrophilia, thrombocytopenia, eosinopenia, and basopenia. ${ }^{7,10-12}$ And the patients with severe disease had more prominent laboratory abnormalities than those with non-severe disease. ${ }^{10}$

Compared with other laboratory tests, the complete blood count (CBC), including the number of white blood cells (WBCs), neutrophils, lymphocytes, is an inexpensive and easy screening technique, and they are sensitive to many pathological changes and may assist in diagnosis when the cause of the disease is unknown. Lymphopenia is a typical laboratory abnormality observed in Severe Acute Respiratory Syndrome Coronavirus (SARS-CoV) ${ }^{13}$ and Middle East Respiratory Syndrome Coronavirus (MERS-CoV) ${ }^{14}$ infections. It's reported to be associated with disease severities. ${ }^{15}$ NLR and LMR are both promptly accessible parameters that can be calculated based on CBC. Elevated NLR leads to poor prognosis. ${ }^{16}$ NLR reflects the balance of the body's innate (neutrophil) and adaptive (lymphocyte) immune responses, ${ }^{17}$ and has prognostic value in various conditions such as cardiovascular disease, ${ }^{18}$ solid tumors, ${ }^{19}$ and Rheumatoid Arthritis, ${ }^{20}$ etc. High monocyte count and low lymphocyte count indicate increased mortality and declined prognosis in a variety of disorders. ${ }^{21}$ It's reported that LMR is a highly sensitive analyzing item used for diagnosis of diabetes, ${ }^{22}$ large B-cell lymphoma, ${ }^{23}$ cardiovascular diseases, ${ }^{21,24,25}$ and COVID$19,{ }^{26}$ and so on.

In this study, we analyzed the data of neutrophil, lymphocyte, monocyte, NLR, and LMR of selected COVID19-infected patients in Chongqing. And we analyzed the associations of blood analyzing items with the different severity groups of COVID-19.

\section{Materials and Methods Data Collection}

We conducted a retrospective study on the clinical features and blood routine results of patients with COVID-19. Routine blood tests were performed using the SYSMEX XE-2100 hematology analyzer and its original matching reagents.

A total of 78 patients infected with SARS-CoV-2 in Chongqing Public Health Medical Center were enrolled from 26 January 2020 to 16 May 2020, of which 61 cases were nonsevere type (including asymptomatic infection, mild and moderate) and 17 cases were severe type (including severe and critical). All cases were defined by the eighth edition diagnosis and treatment plan for COVID-19 issued by the National
Health Commission of the People's Republic of China. The SARS-CoV-2 virus nucleic acid detection of the throat swab of all patients was positive. The groups of COVID-19 patients was performed according to the following guideline: 1) Asymptomatic infection: SARS-CoV-2 virus detection or specific antibody positive but without clinical symptoms; 2) Mild: mild symptoms without pneumonia; 3) Moderate: fever or respiratory tract symptoms with pneumonia; 4) Severe (fulfill any of the three criteria): respiratory distress, respiratory rate $=30$ times $/ \mathrm{min}$; oxygen saturation $=93 \%$ in resting-state; arterial blood oxygen partial pressure $(\mathrm{PaO} 2)$ /oxygen concentration $(\mathrm{FiO} 2)=300 \mathrm{mmHg} ; 5)$ Critical (fulfill any of the three criteria): respiratory failure and require mechanical ventilation; shock incidence; admission to ICU with other organ failures. The first (acute phase), second (progressive phase), and third (convalescent-phase) sample data were collected within one week after symptom onset, two to three weeks, and three to four weeks, respectively. All the clinical records and blood routines of patients were carefully preserved from admission to discharge.

\section{Statistical Analysis}

SPSS software vision 23.0 was used for data statistics. Counts and percentages of categorical variables and continuous quantitative data were represented by median and interquartile ranges (IQR) since non-normally distributed. Proportions for categorical variables were compared using the $\chi 2$ test, and the Fisher exact test was used when the data was limited. Mann-Whitney $U$-test was applied for comparison between two groups. Kruskal-Wallis $\mathrm{H}$ was used for multiple comparisons. ROC curves were constructed for neutrophil, lymphocyte, monocyte, NLR, and LMR during the course of the acute phase. AUC, sensitivity, specificity, positive predictive value, and negative predictive value were calculated and compared. Optimal cut-off values were determined by the Youden index. $\mathrm{P}$ value $<0.05$ was considered statistically significant.

\section{Results \\ Presenting Characteristics}

The study population included 78 laboratory-confirmed patients with COVID-19 in Chongqing. The median age was 45 years (IQR 34.0-55.8), 41 (52.6\%) were female and 37 $(47.4 \%)$ were male. The median days from onset/first nucleic acid positive to admission, admission to discharge, onset/first nucleic acid positive to discharge were 3 days (IQR 2.0-5.0), 21.5 days (IQR 14.3-29.0), 25.0 days (IQR 17.3-32.8), 
respectively. The major clinical symptoms were fever (45 [57.7\%]), cough (41[52.6\%]), expectoration (24[30.8\%]), fatigue (23[29.5\%]), and less common symptoms were diarrhea, chest tightness, headache, pharyngalgia, and rash (6[7.7\%]-19 [24.4\%]). Digestive system diseases (9[11.5\%]) and hypertension $(7[9 \%])$ were the most common basic diseases, while the respiratory system $(20[25.6 \%])$ and digestive system (11 [4.1\%]) were more prone to have comorbidities (Table 1).

Compared with the non-severe group, severe patients were significantly older (median age, 59 years [IQR 51.0 68.0] vs 43 years [IQR 29.0-50.0]; $\mathrm{P}<0.001$ ) and more likely to have potential complications, including respiratory system $(15[88.2 \%]$ vs $5[8.2 \%] ; \mathrm{P}<0.05)$, immune system $(3[17.6 \%]$ vs $1[1.6 \%] ; \mathrm{P}<0.05)$ and skin diseases $(3[17.6 \%]$ vs $0[0.0 \%] ; \mathrm{P}<0.05)$. And the number of days from admission to discharge was also longer in the severe group (27 days [IQR 20.0-35.0] vs 20 days [IQR 13.0 $27.0]$; $\mathrm{P}<0.05$ ). The proportion of symptoms such as headache, fatigue, and chest tightness were increased in severe cases compared with non-severe cases.

The severe patients exhibited lower lymphocyte (1.0 [IQR 0.8-1.3] vs 1.6[IQR 1.2-2.1]; $\mathrm{P}<0.05$ ) but higher NLR (3.3[IQR 2.0-4.3] vs $2.1[1.5-3.0] ; \quad \mathrm{P}<0.05)$. However, there were no significant differences in white blood cell, neutrophil, monocyte, and LMR between the severe and the non-severe group (Table 2).

\section{The Dynamic Profile of Hematological Parameters}

During hospitalization, obvious abnormalities of laboratory blood examinations were found. Lymphocyte and NLR of most patients changed significantly, and the severe group had lower lymphocyte $(\mathrm{P}<0.01)$ but higher NLR $(\mathrm{P}<0.05)$ in the acute, progressive, and convalescentphase. There was a notable difference in the level of neutrophil between non-severe and severe patients in the progressive phase $(\mathrm{P}<0.05)$. And LMR distinguished the non-severe group from the severe group in progressive and convalescent-phase $(\mathrm{P}<0.05)$ (Figure 1).

\section{ROC-Curve Analysis}

To determine the diagnostic utility of hematological indexes within one week after symptom onset, we calculated sensitivity, specificity, positive predictive value, and negative predictive value between the two groups using the ROC curves (Table 3). Among the five parameters (lymphocyte, NLR, monocyte, LMR, and neutrophil), the highest AUC was lymphocyte (0.74) with an optimal cutoff of 1.14 , the sensitivity of $70.59 \%$, specificity of $80.33 \%$, the positive predictive value of $50.00 \%$, and negative predictive value of $90.74 \%$. At a cut-off of 2.36, the corresponding sensitivity, specificity, positive predictive value, negative predictive value, and AUC of the NLR were $76.47 \%, 67.21 \%, 39.39 \%, 91.11 \%$, and 0.67 . At a cut-off of 0.25 , the sensitivity, specificity, positive predictive value, negative predictive value, and AUC of monocyte were $35.29 \%, 95.08 \%, 66.67 \%, 84.06 \%$, and 0.65. However, the AUC of LMR and neutrophil were only 0.57 and 0.50 , respectively (Figure 2).

\section{Discussion}

The human-to-human transmission of SARS-CoV-2 among close contacts was beyond imagination. ${ }^{27} \mathrm{An}$ indepth understanding of the characteristics of COVID-19 is crucial for its early prevention and treatment. To explore the characteristics of COVID-19 patients during their hospitalization, 78 COVID-19 patients in Chongqing were included for further study.

The common symptoms of Chongqing COVID-19 on admission were fever, cough, expectoration, and fatigue. These performances are consistent with those reported in several studies. ${ }^{3,28,29}$ Nevertheless, fever, chills, malaise, and myalgia were major symptoms of SARS-CoV, ${ }^{30}$ while the common symptoms of MERS-CoV were fever, cough, dyspnea, and chest pain. ${ }^{31}$ In this study, we discovered that digestive system diseases and high blood pressure were the most common underlying diseases, and the majority of complications tended to occur in the respiratory and digestive systems. In severe cases, age, as well as the proportion of underlying diseases, were higher than in non-severe cases coinciding with the results of a few studies, ${ }^{3,8,10}$ which means that age and comorbidities may be risk factors for adverse outcomes. However, there is no obvious difference between the sexes. This phenomenon was different from the recent report showing that COVID-19 was more likely to affect men. ${ }^{7}$ The possible explanation was that majority of the first batch of people who came into contact with the Seafood Wholesale Market in southern China were male workers.

The dynamic profile of hematological parameters was monitored in 78 COVID-19 patients. Hematological items of patients with different severity were compared, and five parameters (lymphocyte, NLR, monocyte, LMR, and neutrophil) were selected for the ROC analyzing. Asghar et al found an NLR value of 5.48 and LMR value of 2.85 admitted in an intensive care unit (ICU) as the potential markers for 
Table I Clinical Characteristic of COVID-19 Patients in Chongqing

\begin{tabular}{|c|c|c|c|c|}
\hline & Total $(\mathbf{N}=\mathbf{7 8})$ & Non-Severe $(\mathbf{N}=6 \mathrm{I})$ & Severe $(N=17)$ & P value \\
\hline \multicolumn{5}{|l|}{ Sex-n. (\%) } \\
\hline Male & $37(47.4)$ & $27(44.3)$ & $10(58.8)$ & 0.288 \\
\hline Female & $4 \mid(52.6)$ & $34(55.7)$ & $7(4 I .2)$ & \\
\hline Age-year & $45.5(34.0-55.8)$ & $43.0(29.0-50.0)$ & $59.0(51.0-68.0)$ & 0.000 \\
\hline Smoke-no. (\%) & $17(2 \mid .8)$ & $13(2 \mid .3)$ & $4(23.5)$ & 0.845 \\
\hline Alcohol-no. (\%) & $21(26.9)$ & $14(23.0)$ & $7(4 \mid .2)$ & 0.234 \\
\hline Days from Onset/first nucleic acid positive to Admission & $3.0(2.0-5.0)$ & $3.0(2.0-4.0)$ & $5.0(4.0-6.0)$ & 0.001 \\
\hline Days from Admission to Discharge & $21.5(14.3-29.0)$ & $20.0(13.0-27.0)$ & $27.0(20.0-35.0)$ & 0.019 \\
\hline Days from Onset/first nucleic acid positive to Discharge & $25.0(17.3-32.8)$ & $22.0(17.0-29.0)$ & $31.0(26.0-37.0)$ & 0.001 \\
\hline Acute Phase & $4.0(3.0-6.0)$ & $4.0(3.0-5.0)$ & $7.0(5.0-7.0)$ & 0.000 \\
\hline Progressive Phase & $11.0(9.0-13.0)$ & II.0 (9.0-12.0) & $13.0(12.0-14.0)$ & 0.002 \\
\hline Convalescent Phase & $24.5(17.0-29.0)$ & $22.0(16.0-29.0)$ & $27.0(25.0-29.0)$ & 0.021 \\
\hline \multicolumn{5}{|l|}{ Symptom-no. (\%) } \\
\hline Fever & $45(57.7)$ & $29(47.5)$ & $16(94.1)$ & 0.001 \\
\hline Cough & $41(52.6)$ & $27(44.3)$ & $14(82.4)$ & 0.005 \\
\hline Expectoration & $24(30.8)$ & $15(24.6)$ & $9(52.9)$ & 0.025 \\
\hline Pharyngalgia & II (I4.I) & $10(16.4)$ & I (5.9) & 0.479 \\
\hline Headache & $15(19.2)$ & 7 (II.5) & $8(47.1)$ & 0.003 \\
\hline Fatigue & $23(29.5)$ & $10(16.4)$ & $13(76.5)$ & 0.000 \\
\hline Diarrhea & $19(24.4)$ & $14(23.0)$ & $5(29.4)$ & 0.819 \\
\hline Chest tightness & $16(20.5)$ & 7 (II.5) & $9(52.9)$ & 0.001 \\
\hline Rash & $6(7.7)$ & $3(4.9)$ & $3(17.6)$ & 0.220 \\
\hline \multicolumn{5}{|l|}{ Basic diseases-no. (\%) } \\
\hline Hypertension & $7(9.0)$ & $5(8.2)$ & $2(11.8)$ & 1.000 \\
\hline Diabetes & $5(6.4)$ & $0(0.0)$ & $5(29.4)$ & 0.000 \\
\hline Hematological System diseases & $4(5.1)$ & $2(3.3)$ & $2(11.8)$ & 0.435 \\
\hline Digestive system diseases & $9(11.5)$ & $6(9.8)$ & $3(17.6)$ & 0.644 \\
\hline Cardiovascular system diseases & $3(3.8)$ & I (I.6) & $2(11.8)$ & 0.228 \\
\hline Respiratory system diseases & $\mathrm{I}(\mathrm{I} .3)$ & $0(0.0)$ & I (5.9) & 0.492 \\
\hline Other Metabolic diseases & $3(3.8)$ & $2(3.3)$ & I (5.9) & 1.000 \\
\hline \multicolumn{5}{|l|}{ Comorbidity-no. (\%) } \\
\hline Respiratory system & $20(25.6)$ & $5(8.2)$ & I5 (88.2) & 0.000 \\
\hline Digestive system & II (4.I) & 7 (II.5) & $4(23.5)$ & 0.385 \\
\hline Metabolic diseases & $2(2.6)$ & $2(3.3)$ & $0(0.0)$ & 1.000 \\
\hline Hematological System & $5(6.4)$ & $4(6.6)$ & I (5.9) & 1.000 \\
\hline Immune system & $4(5.1)$ & $\mathrm{I}(\mathrm{I} .6)$ & $3(17.6)$ & 0.043 \\
\hline Urinary system & $\mathrm{I}(\mathrm{I} .3)$ & $0(0.0)$ & I (5.9) & 0.492 \\
\hline Neuropsychological system & $2(2.6)$ & I (I.6) & I (5.9) & 0.911 \\
\hline Skin diseases & $3(3.8)$ & $0(0.0)$ & $3(17.6)$ & 0.008 \\
\hline Cardiovascular system & $2(2.6)$ & I (I.6) & I (5.9) & 0.911 \\
\hline Other diseases & $4(5.1)$ & I (I.6) & $3(17.6)$ & 0.043 \\
\hline \multicolumn{5}{|l|}{ Chest CT-no. (\%) } \\
\hline Chest radiographs abnormalities & $59(75.6)$ & $42(68.9)$ & $17(100.0)$ & 0.020 \\
\hline
\end{tabular}


Table 2 The Correlation of Hematological Parameters During the Course of Acute Phase with the Severity of COVID-19 in Chongqing

\begin{tabular}{|l|l|l|l|l|}
\hline Baseline Variables & Total $(\mathbf{N}=\mathbf{7 8})$ & Non-Severe $(\mathbf{N}=6 \mathbf{I})$ & Severe $(\mathbf{N}=\mathbf{I 7})$ & P value \\
\hline WBC $\left(10^{9} / \mathrm{L}\right)$ & $5.3(4.2-6.7)$ & $5.4(4.2-6.8)$ & $4.9(3.9-5.9)$ & 0.189 \\
Neutrophil $\left(10^{9} / \mathrm{L}\right)$ & $3.1(2.4-4.4)$ & $3.2(2.2-4.4)$ & $3.0(2.7-4.4)$ & 0.961 \\
Lymphocyte $\left(10^{9} / \mathrm{L}\right)$ & $1.4(1.0-2.0)$ & $1.6(1.2-2.1)$ & $1.0(0.8-1.3)$ & 0.003 \\
Monocyte $\left(10^{9} / \mathrm{L}\right)$ & $0.4(0.3-0.5)$ & $0.4(0.3-0.5)$ & $0.3(0.2-0.5)$ & 0.059 \\
NLR & $2.2(1.6-3.3)$ & $2.1(1.5-3.0)$ & $3.3(2.0-4.3)$ & 0.028 \\
LMR & $3.7(2.6-4.8)$ & $3.8(2.7-5.4)$ & $3.7(2.5-4.3)$ & 0.374 \\
\hline
\end{tabular}

Abbreviations: NLR, neutrophil-lymphocyte ratio; LMR, lymphocyte-monocyte ratio.

severity of disease, with AUC of 0.841 and $0.227 .^{32}$ In our study, we concluded that a slightly lower AUC of NLR was 0.67 at a cut-off value of 2.36 while a relatively higher AUC of LMR was 0.57 at a cut-off value of 4.75 . The risk factors, age, and gender of the distinct populations included in the two studies were different, which may account for this phenomenon. And the AUC of lymphocyte, monocyte, and neutrophil were $0.74,0.65$, and 0.50 , respectively, which were generally higher than that of another study. ${ }^{33}$

Recent reports have shown that lymphocyte counts are normal in COVID-19 patients with mild disease. In contrast, $20-96.1 \%$ of severe diseases have lymphopenia. ${ }^{10,34}$ Similarly, we found that the severe group had progressively decreased lymphocyte count during hospitalization. With the application of NLR to COVID-19, elevated NLR on admission was considered an independent risk factor for severe disease and poor clinical outcomes in COVID-19 patients. $^{35,36}$ Similarly, the higher level of NLR during hospitalization in severe patients in this study also suggested an association of NLR with disease severity and clinical course. The result of the level of neutrophil in the progressive phase was higher but LMR was lower in the progressive and convalescent-phase in severe patients coincided with previous studies. ${ }^{32,37,38}$ In summary, lymphocyte, and NLR showed unexceptionable performance in patients' courses which could distinguish the severe group from the nonsevere group.

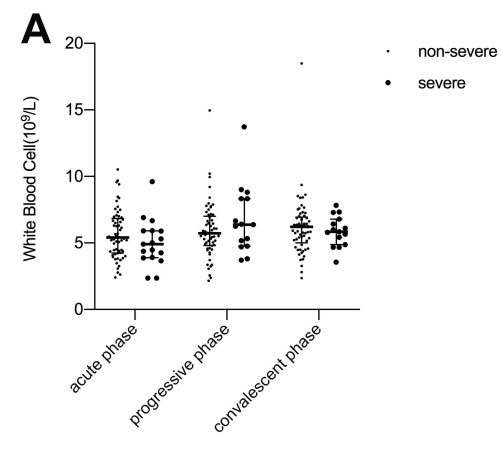

D

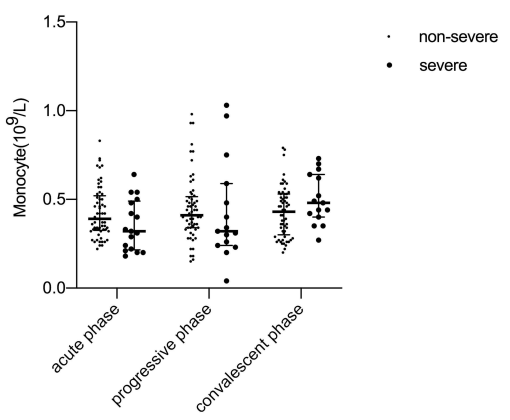

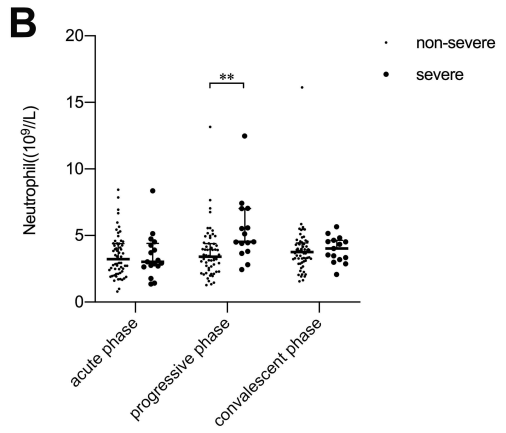

E

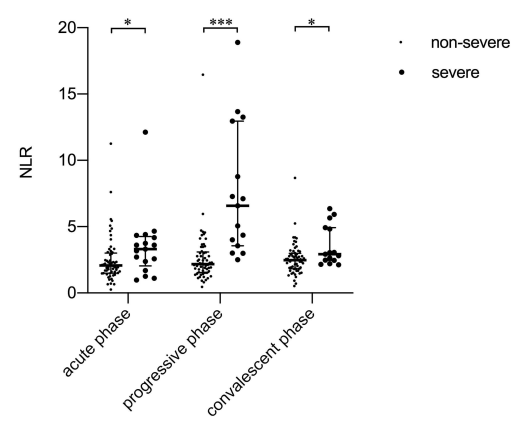

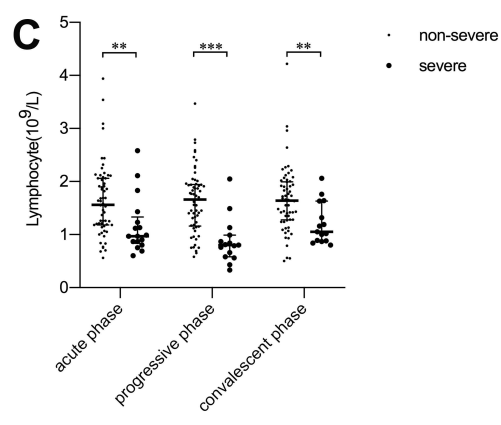

$\mathbf{F}$

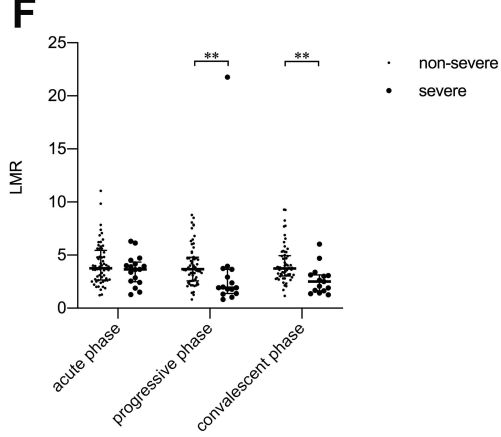

Figure I Dynamic changes of blood analyzing items of COVID-19 between the severe and non-severe groups (A-F). The levels of white blood cell (A), neutrophil (B), lymphocyte (C), monocyte (D), NLR (E), and LMR (F) on three different phases (the acute, progressive, and convalescent-phase) between the severe and non-severe group. $* \mathrm{P}<0.05$, $* * \mathrm{P}<0.01$, $* * * \mathrm{P}<0.001$. 
Table 3 The Value of Lymphocyte, NLR, Monocyte, LMR, and Neutrophil in the Diagnosis of COVID-19

\begin{tabular}{|l|c|c|c|c|c|}
\hline Item & Sensitivity (\%) & Specificity (\%) & Positive Predictive Value (\%) & Negative Predictive Value (\%) & Cut-off Value \\
\hline Lymphocyte & 70.59 & 80.33 & 50.00 & 90.74 & 1.14 \\
NLR & 76.47 & 67.21 & 39.39 & 91.11 & 2.36 \\
Monocyte & 35.29 & 95.08 & 66.67 & 84.06 & 0.25 \\
LMR & 88.24 & 32.79 & 26.79 & 90.91 & 4.75 \\
Neutrophil & 82.35 & 36.07 & 26.42 & 88.00 & 2.64 \\
\hline
\end{tabular}

The purpose of our study is not only to provide hematological markers for reference but also to alert the clinicians to notice active surveillance for COVID-19 cases. And there were some limitations: as a single-center study, these results should be interpreted with caution owing to the small sample size of this observational study, bias and residual confusion may occur. Multi-center and large-scale researches are needed to further define the clinical implication of dynamic hematological parameters in COVID-19 cases.

\section{Conclusion}

In general, our results showed the hematological features of COVID-19 cases in Chongqing. There were remarkable differences in blood routine analyzing items including lymphocyte, neutrophil, LMR, and NLR in COVID-19 patients. And the dynamic changes of lymphocyte and neutrophil-lymphocyte ratio during the course of the disease may be useful for discriminating the severe group and non-severe group.

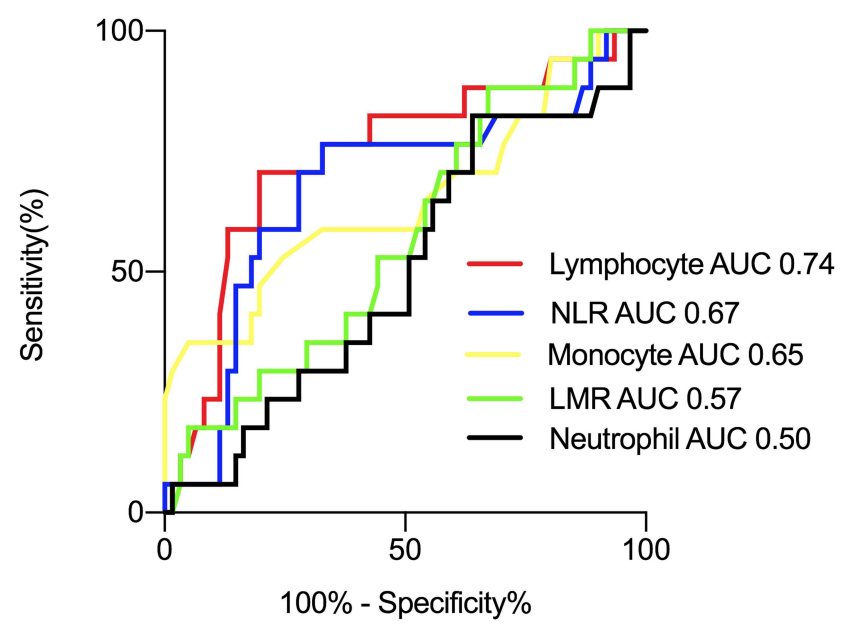

Figure 2 The receiver operation curves of hematological parameters for disease severity classification of COVID-19. ROC analysis of lymphocyte, monocyte, NLR, LMR, and neutrophil for discriminating 17 severe cases from 61 non-severe cases.

\section{Ethical Approval}

The study was approved by Chongqing General Hospital Ethics Committee [S2020-021-01]. Due to the nature of this retrospective study without any additional interventions, the need for consent was waived by the ethics committee. All personally identifiable records were kept confidential. The guidelines outlined in the Declaration of Helsinki were followed in this study.

\section{Acknowledgments}

This study was funded by Chongqing Municipal Health and Health Committee, Chongqing Science and Technology Bureau (2020FYYX169). We thank all patients involved in this study.

\section{Disclosure}

The authors declare that there is no conflict of interests.

\section{References}

1. May M, Rostama B, Relich RF. Selectomic and evolvability analyses of the highly pathogenic betacoronaviruses SARS-CoV-2, SARS-CoV, and MERS-CoV. 2020. doi:10.1101/2020.05.05.078956

2. Wang D, Hu B, Hu C, et al. Clinical characteristics of 138 hospitalized patients with 2019 novel coronavirus-infected pneumonia in Wuhan, China. JAMA. 2020;323(11):1061-1069. doi:10.1001/ jama.2020.1585

3. Song JW, Zhang C, Fan X, et al. Immunological and inflammatory profiles in mild and severe cases of COVID-19. Nat Commun. 2020;11 (1):3410. doi:10.1038/s41467-020-17240-2

4. Wu C, Chen X, Cai Y, et al. Risk factors associated with acute respiratory distress syndrome and death in patients with coronavirus disease 2019 pneumonia in Wuhan, China. JAMA Intern Med. 2020;180(7):934-943. doi:10.1001/jamainternmed.2020.0994

5. Wan S, Yi Q, Fan S, et al. Relationships among lymphocyte subsets, cytokines, and the pulmonary inflammation index in coronavirus (COVID-19) infected patients. Br J Haematol. 2020;189(3):428-437. doi:10.1111/bjh.16659

6. Zhang G, Zhang J, Wang B, Zhu X, Wang Q, Qiu S. Analysis of clinical characteristics and laboratory findings of 95 cases of 2019 novel coronavirus pneumonia in Wuhan, China: a retrospective analysis. Respir Res. 2020;21(1):74. doi:10.1186/s12931-02001338-8

7. Chen N, Zhou M, Dong X, et al. Epidemiological and clinical characteristics of 99 cases of 2019 novel coronavirus pneumonia in Wuhan, China: a descriptive study. Lancet. 2020;395 (10223):507-513. doi:10.1016/S0140-6736(20)30211-7 
8. Liu J, Li S, Liu J, et al. Longitudinal characteristics of lymphocyte responses and cytokine profiles in the peripheral blood of SARS-CoV-2 infected patients. EBioMedicine. 2020;55:102763. doi:10.1016/j.ebiom.2020.102763

9. Fu J, Kong J, Wang W, et al. The clinical implication of dynamic neutrophil to lymphocyte ratio and D-dimer in COVID-19: a retrospective study in Suzhou China. Thromb Res. 2020;192:3-8. doi:10.1016/j.thromres.2020.05.006

10. Guan WJ, Ni ZY, Hu Y, et al. Clinical characteristics of coronavirus disease 2019 in China. $N$ Engl $J$ Med. 2020;382(18):1708-1720. doi:10.1056/NEJMoa2002032

11. Fan BE, Chong VCL, Chan SSW, et al. Hematologic parameters in patients with COVID-19 infection. Am J Hematol. 2020;95(6):e131e134. doi:10.1002/ajh.25774

12. Qin $\mathrm{C}$, Zhou L, Hu Z, et al. Dysregulation of immune response in patients with coronavirus 2019 (COVID-19) in Wuhan, China. Clin Infect Dis. 2020;71(15):762-768. doi:10.1093/cid/ciaa248

13. Booth CM, Matukas LM, Tomlinson GA, et al. Clinical features and short-term outcomes of 144 patients with SARS in the greater Toronto area. JAMA. 2003;289(21):2809. doi:10.1001/jama.289.21. JOC30885

14. Assiri A, Al-Tawfiq JA, Al-Rabeeah AA, et al. Epidemiological, demographic, and clinical characteristics of 47 cases of Middle East respiratory syndrome coronavirus disease from Saudi Arabia: a descriptive study. Lancet Infect Dis. 2013;13(9):752-761. doi:10.1016/S1473-3099(13)70204-4

15. de Wit E, van Doremalen N, Falzarano D, Munster VJ. SARS and MERS: recent insights into emerging coronaviruses. Nat Rev Microbiol. 2016;14(8):523-534. doi:10.1038/nrmicro.2016.81

16. Ni J, Wang H, Li Y, Shu Y, Liu Y. Neutrophil to lymphocyte ratio (NLR) as a prognostic marker for in-hospital mortality of patients with sepsis: a secondary analysis based on a single-center, retrospective, cohort study. Medicine (Baltimore). 2019;98(46):e18029. doi:10.1097/MD.0000000000018029

17. Azab B, Camacho-Rivera M, Taioli E. Average values and racial differences of neutrophil lymphocyte ratio among a nationally representative sample of United States subjects. PLoS One. 2014;9(11): e112361. doi:10.1371/journal.pone. 0112361

18. Kim S, Eliot M, Koestler DC, Wu WC, Kelsey KT. Association of neutrophil-to-lymphocyte ratio with mortality and cardiovascular disease in the Jackson heart study and modification by the Duffy antigen variant. JAMA Cardiol. 2018;3(6):455. doi:10.1001/jamacardio.2018.1042

19. Templeton AJ, McNamara MG, Šeruga B, et al. Prognostic role of neutrophil-to-lymphocyte ratio in solid tumors: a systematic review and meta-analysis. J Natl Cancer Inst. 2014;106(6):dju124. doi:10.1093/jnci/dju124

20. Targonska-Stepniak B, Zwolak R, Piotrowski M, Grzechnik K, Majdan M. The relationship between hematological markers of systemic inflammation (Neutrophil-To-Lymphocyte, Platelet-To-Lymphocyte, Lymphocyte-To-Monocyte Ratios) and ultrasound disease activity parameters in patients with rheumatoid arthritis. J Clin Med. 2020;9(9):2760. doi: $10.3390 / \mathrm{jcm} 9092760$

21. Balta S, Demırer Z, Aparci M, Yildirim AO, Ozturk C. The lymphocyte-monocyte ratio in clinical practice. J Clin Pathol. 2016;69(1):88-89. doi:10.1136/jclinpath-2015-203233

22. Demirdal T, Sen P. The significance of neutrophil-lymphocyte ratio, platelet-lymphocyte ratio and lymphocyte-monocyte ratio in predicting peripheral arterial disease, peripheral neuropathy, osteomyelitis and amputation in diabetic foot infection. Diabetes Res Clin Pract. 2018;144:118-125. doi:10.1016/j.diabres.2018.08.009
23. Bento L, Díaz-López A, Barranco G, et al. New prognosis score including absolute lymphocyte/monocyte ratio, red blood cell distribution width and beta- 2 microglobulin in patients with diffuse large B-cell lymphoma treated with R-CHOP: Spanish Lymphoma Group Experience (GELTAMO). Br J Haematol. 2020;188(6):888-897. doi:10.1111/bjh. 16263

24. Gong S, Gao X, Xu F, et al. Association of lymphocyte to monocyte ratio with severity of coronary artery disease. Medicine (Baltimore). 2018;97(43):e12813. doi:10.1097/MD.0000000000012813

25. Chen H, Li M, Liu L, Dang X, Zhu D, Tian G. Monocyte/lymphocyte ratio is related to the severity of coronary artery disease and clinical outcome in patients with non-ST-elevation myocardial infarction. Medicine (Baltimore). 2019;98(26):e16267. doi:10.1097/MD.0000000000016267

26. Lissoni P, Rovelli F, Monzon A, et al. Evidence of abnormally low lymphocyte-to-monocyte ratio in covid-19-induced severe acute respiratory syndrome. J Immunol Allergy. 2020;1(2):1-6.

27. Phan LT, Nguyen TV, Luong QC, et al. Importation and human-tohuman transmission of a novel coronavirus in Vietnam. $N$ Engl $J$ Med. 2020;382(9):872-874. doi:10.1056/NEJMc2001272

28. Wan S, Xiang Y, Fang W, et al. Clinical features and treatment of COVID-19 patients in northeast Chongqing. J Med Virol. 2020;92 (7):797-806. doi:10.1002/jmv.25783

29. Li R, Tian J, Yang F, et al. Clinical characteristics of 225 patients with COVID-19 in a tertiary hospital near Wuhan, China. J Clin Virol. 2020;127:104363. doi:10.1016/j.jcv.2020.104363

30. Liu CL, Lu YT, Peng MJ, et al. Clinical and laboratory features of severe acute respiratory syndrome vis-a-vis onset of fever. Chest. 2004;126(2):517. doi:10.1378/chest.126.2.509

31. Al-Abdallat MM, Payne DC, Alqasrawi S, et al. Hospital-associated outbreak of Middle East respiratory syndrome coronavirus: a serologic, epidemiologic, and clinical description. Clin Infect Dis. 2014;59(9):1225-1233. doi:10.1093/cid/ciu359

32. Asghar MS, Khan NA, Haider Kazmi SJ, et al. Hematological parameters predicting severity and mortality in COVID-19 patients of Pakistan: a retrospective comparative analysis. J Community Hosp Intern Med Perspect. 2020;10(6):514-520. doi:10.1080/ 20009666.2020.1816276

33. Asghar MS, Haider Kazmi SJ, Ahmed Khan N, et al. Clinical profiles, characteristics, and outcomes of the first 100 admitted COVID-19 patients in Pakistan: a single-center retrospective study in a tertiary care hospital of Karachi. Cureus. 2020;12(6):e8712. doi: $10.7759 /$ cureus. 8712

34. Ding X, Yu Y, Lu B, et al. Dynamic profile and clinical implications of hematological parameters in hospitalized patients with coronavirus disease 2019. Clin Chem Lab Med. 2020;58(8):1365-1371. doi:10.1515/cclm-2020-0411

35. Yang AP, Liu JP, Tao WQ, Li HM. The diagnostic and predictive role of NLR, d-NLR and PLR in COVID-19 patients. Int Immunopharmacol. 2020;84:106504. doi:10.1016/j.intimp.2020.106504

36. Liu Y, Du X, Chen J, et al. Neutrophil-to-lymphocyte ratio as an independent risk factor for mortality in hospitalized patients with COVID-19. $J$ Infect. 2020;81(1):e6-e12. doi:10.1016/j. jinf.2020.04.002

37. Huang J, Cheng A, Lin S, Zhu Y, Chen G. Individualized prediction nomograms for disease progression in mild COVID-19. J Med Virol. 2020;92(10):2074-2080. doi:10.1002/jmv.25969

38. Qian GQ, Yang NB, Ding F, et al. Epidemiologic and clinical characteristics of 91 hospitalized patients with COVID-19 in Zhejiang, China: a retrospective, multi-centre case series. Qjm. 2020;113 (7):474-481. doi:10.1093/qjmed/hcaa089 


\section{Publish your work in this journal}

The International Journal of General Medicine is an international, peer-reviewed open-access journal that focuses on general and internal medicine, pathogenesis, epidemiology, diagnosis, monitoring and treatment protocols. The journal is characterized by the

across all disease areas. The manuscript management system is completely online and includes a very quick and fair peer-review system, which is all easy to use. Visit http://www.dovepress.com/ rapid reporting of reviews, original research and clinical studies testimonials.php to read real quotes from published authors. 